\title{
Construction of Codes Identifying Sets of Vertices
}

\author{
Sylvain Gravier \\ CNRS - UJF, ERTé "Maths à Modeler", Groupe de Recherche GéoD \\ Laboratoire Leibniz, 46, avenue Félix Viallet, 38031 Grenoble Cedex (France) \\ sylvain.gravier@imag.fr \\ Julien Moncel \\ CNRS - UJF, ERTé "Maths à Modeler", Groupe de Recherche GéoD \\ Laboratoire Leibniz, 46, avenue Félix Viallet, 38031 Grenoble Cedex (France) \\ julien.moncel@imag.fr
}

Submitted: Feb 8, 2005; Accepted: Mar 1, 2005; Published: Mar 8, 2005

Mathematics Subject Classifications: 05C99, 94B60, 94C12

\begin{abstract}
In this paper the problem of constructing graphs having a $(1, \leq \ell)$-identifying code of small cardinality is addressed. It is known that the cardinality of such a code is bounded by $\Omega\left(\frac{\ell^{2}}{\log \ell} \log n\right)$. Here we construct graphs on $n$ vertices having a $(1, \leq \ell)$-identifying code of cardinality $O\left(\ell^{4} \log n\right)$ for all $\ell \geq 2$. We derive our construction from a connection between identifying codes and superimposed codes, which we describe in this paper.
\end{abstract}

\section{Codes identifying sets of vertices}

Let $G=(V, E)$ be a simple, non-oriented graph. For a vertex $v \in V$, let us denote by $N[v]$ the closed neighborhood of $v: N[v]=N(v) \cup\{v\}$. Let $C \subseteq V$ be a subset of vertices of $G$, and for all nonempty subset of at most $\ell$ vertices $X \subseteq V$, let us denote

$$
I(X)=I(X, C):=\bigcup_{x \in X} N[x] \cap C .
$$

If all the $I(X, C)$ 's are distinct, then we say that $C$ separates the sets of at most $\ell$ vertices of $G$, and if all the $I(X, C)$ 's are nonempty then we say that $C$ covers the sets of at most $\ell$ vertices of $G$. We say that $C$ is a code identifying sets of at most $\ell$ vertices of $G$ if and only if $C$ covers and separates all the sets of at most $\ell$ vertices of $G$. The dedicated terminology [12] for such codes is $(1, \leq \ell)$-identifying codes. The sets $I(X)$ are said to be the identifying sets of the corresponding $X$ 's. 
Whereas $C=V$ is trivially always a code covering the sets of at most $\ell$ vertices of any graph $G=(V, E)$, not every graph has a $(1, \leq \ell)$-identifying code. For example, if $G$ contains two vertices $u$ and $v$ such that $N[u]=N[v]$, then $G$ has no $(1, \leq \ell)$-identifying code, since for any subset of vertices $C$ we have $N[u] \cap C=N[v] \cap C$. Actually, a graph admits a $(1, \leq \ell)$-identifying code if and only if for every pair of subsets $X \neq Y$, $|X|,|Y| \leq \ell$, we have $N[X] \neq N[Y]$, where $N[X]$ denotes $\bigcup_{x \in X} N[x]$. In the case where $G$ admits a $(1, \leq \ell)$-identifying code, then $C=V$ is always a $(1, \leq \ell)$-identifying code of $G$, hence we are usually interested in finding a $(1, \leq \ell)$-identifying code of minimum cardinality.

These codes are used for fault diagnosis in multiprocessor systems, and were first defined in [9]. The problem of constructing such codes has already been addressed in $[1,2,12,9,10,7]$. In these papers the authors used covering codes, that are quite well known [3]. We refer the reader to [14] for an online up-to date bibliography about identifying codes.

In the general case $\ell \geq 1$, another good framework to construct such codes is to use $\ell$-superimposed codes, as suggested in [6]. Indeed, given a graph $G=(V, E)$ together with a $(1, \leq \ell)$-identifying code $C$ of $G$, the characteristic vectors of the subsets $I(X, C)$, for $|X| \leq \ell$, satisfy the following property :

The boolean sum (OR) of any set of at most $\ell$ vectors is distinct from the boolean sum of any other set of at most $\ell$ vectors.

A set of vectors satisfying $(1)$ is a $U D_{\ell}$-code, or $\ell$-superimposed code. These codes were defined by Kautz and Singleton in [11], and about such codes we know the following :

Theorem 1 Let $K$ be a maximum $\ell$-superimposed code of $\{0,1\}^{N}$. Then there exist two constants $c_{1}$ and $c_{2}$, not depending on $N$ or $\ell$, such that

$$
2^{c_{1} N / \ell^{2}} \leq|K| \leq 2^{c_{2} N \log \ell / \ell^{2}} .
$$

Moreover the lower bound is constructive : there exists an algorithm which, given $N$ and $\ell$, constructs an $\ell$-superimposed code of $\{0,1\}^{N}$ of cardinality $2^{c_{1} N / \ell^{2}}$.

The lower bound comes from [11], and a combinatorial proof of the upper bound, originally established in [4], can be found, for example, in [13]. A greedy algorithm constructing an $\ell$-superimposed code of cardinality $2^{c_{1} N / \ell^{2}}$ can be found in [8].

It was already explained in [6] that it was easy to get an $\ell$-superimposed code from a $(1, \leq \ell)$-identifying code. In this paper we show that we can also get a $(1, \leq \ell)$-identifying code from an $\ell$-superimposed code, which answers to a question of [6]. We give such a construction and prove the following :

Theorem 2 For all $\ell \geq 1$, there exists a function $c(n)=O\left(\ell^{4} \log n\right)$ and an infinite family of graphs $\left(G_{i}\right)_{i \in \mathbb{N}}$, such that, for all $i \in \mathbb{N}, G_{i}$ has $n_{i}$ vertices and admits a $(1, \leq \ell)$ identifying code of cardinality $c\left(n_{i}\right)$, with $n_{i} \rightarrow \infty$ when $i \rightarrow \infty$. Moreover we can explicitly construct such a family of graphs $\left(G_{i}\right)_{i \in \mathbb{N}}$. 
In the next section we describe our construction. In section 3 we show the validity of our construction, which proves Theorem 2. In the last section, we give an open problem connected to our construction.

\section{Construction of Identifying Codes}

Let $\ell \geq 2$. In this section we describe the construction of a graph $\mathcal{G}$ together with a $(1, \leq \ell)$-identifying code $C$ of $\mathcal{G}$. Its validity is proved in the next section.

1. Let $N=\left\lceil\ell^{2} \log n\right\rceil$ and let $K$ be a maximal $\ell$-superimposed code of $\{0,1\}^{N}$, that is to say there is no $K^{\prime} \supset K, K^{\prime} \neq K$, such that $K^{\prime}$ is an $\ell$-superimposed code. Let $k$ denote the cardinality of $K: K=V_{1}, \ldots, V_{k}$.

2. Consider the $N \times k$ matrix $M$ whose columns are the vectors of $K$. Let $M^{\prime}$ be a $N \times N$ submatrix of $M$ such that there is a 1 on every row of $M^{\prime}$.

3. Let $H$ be a connected graph admitting a $(1, \leq \ell)$-identifying code. From $M$ and $M^{\prime}$, let us construct a graph $\mathcal{G}=G\left(M, M^{\prime}\right)$ together with $C=C\left(M, M^{\prime}\right)$ a $(1, \leq \ell)$ identifying code of $\mathcal{G}$ as follows. The subgraph induced by the code $\mathcal{G}[C]$ consists in the disjoint union of $N$ copies of $H$. In each copy $H_{i}$ of $H$ we specify one vertex $h_{i}, i=1, \ldots, N$. These vertices $h_{1}, \ldots, h_{N}$ will be such that

$$
N(V(\mathcal{G}) \backslash C)=\left\{h_{1}, \ldots, h_{N}\right\} .
$$

Now, to each column $V_{j}$ of $M \backslash M^{\prime}$ we associate a vertex $v_{j}=\phi\left(V_{j}\right)$ of $\mathcal{G}$, whose neighbors are the $h_{i}$ 's for each $i$ such that the $i$-th coordinate of $V_{j}$ is equal to 1 (see Figure 1). There are no edges between the $v_{j}$ 's, hence $V_{j}$ is the characteristic vector of the identifying set of $v_{j}$, which is also the neighborhood of $v_{j}$.

\section{Proof of the validity of the construction}

We show the validity of the construction described in the previous section and we prove Theorem 2. In Step 2 of the construction, we needed the following:

Lemma 1 Let $M$ be an $n \times m(n \leq m) 0$-1-matrix which has no row consisting only of 0 's. Then there exists an $n \times n^{\prime}\left(n^{\prime} \leq n\right)$ submatrix $M^{\prime}$ of $M$ such that there is a 1 on every row of $M^{\prime}$.

Proof : Let $M$ be a matrix satisfying the requirements of the lemma. Let $M_{1}, \ldots, M_{m}$ be the columns of $M$.

The proof works by induction on $n$. Without loss of generality, we may assume that there exists $p \leq n$ such that $M_{i, 1}=1$ for all $i \leq p$ and $M_{j, 1}=0$ for all $j>p$. If $p=n$ then the lemma holds. Otherwise, let $P$ be the matrix consisting in the restriction of the 


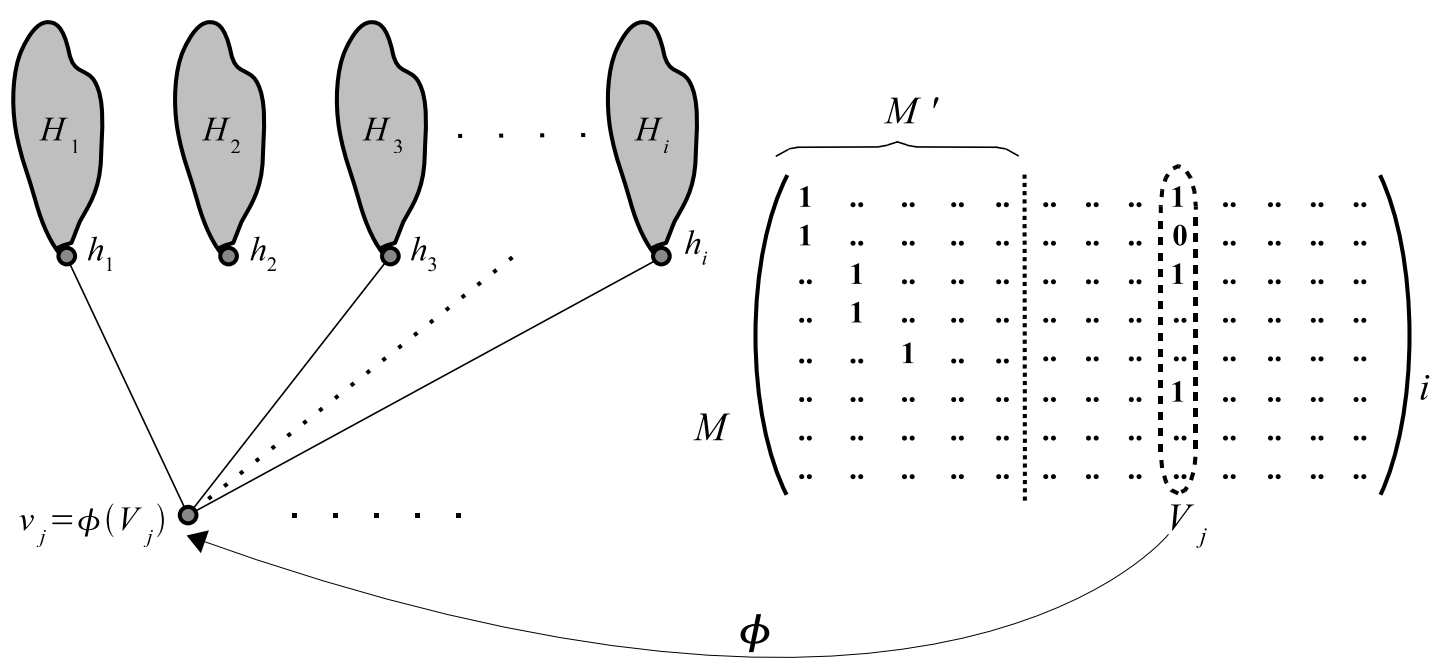

Figure 1: Construction of a graph $\mathcal{G}=\mathcal{G}\left(M, M^{\prime}\right)$ together with a $(1, \leq \ell)$ - identifying code $C=C\left(M, M^{\prime}\right)$ of $\mathcal{G}$ from $M$ and $M^{\prime}$.

columns $M_{2}, \ldots, M_{m}$ to the rows indexed by $p+1, \ldots, n$. By induction, there exists a submatrix $P^{\prime}$ of $P$ such that there is a 1 on every row of $P^{\prime}$. Now, the submatrix $M^{\prime}$ of $M$ defined by the columns of $P^{\prime}$ plus $M_{1}$ satisfies the requirement.

Since a matrix of a maximal $\ell$-superimposed code of $\{0,1\}^{N}$ is a $0-1$-matrix with no row consisting only of 0 's, we get, by the previous lemma :

Lemma 2 Let $M$ be an $N \times k$ matrix whose columns are the vectors of a maximal $\ell$ superimposed code of $\{0,1\}^{N}$. Then there exists an $N \times N^{\prime}\left(N^{\prime} \leq N\right)$ submatrix $M^{\prime}$ of $M$ such that there is a 1 on every row of $M^{\prime}$.

Later we will also need the following :

Lemma 3 Let $M$ be an $N \times k$ matrix whose columns are the vectors of $K$, a maximal $\ell$-superimposed code of $\{0,1\}^{N}$, and let $M^{\prime}$ be an $N \times N^{\prime}\left(N^{\prime} \leq N\right)$ submatrix of $M$ such that there is a 1 on every row of $M^{\prime}$ (by the previous Lemma such a submatrix exists). Then every column of $M \backslash M^{\prime}$ has at least $\ell$ nonzero coordinates.

Proof : Let $V$ be a column of $M \backslash M^{\prime}$ having less than $\ell$ nonzero coordinates. Since there is a 1 on every row of $M^{\prime}$ then we can find $\left\{V_{1}, \ldots, V_{m}\right\}, m \leq \ell-1$, a set of at most $\ell-1$ columns of $M^{\prime}$, such that

$$
V \leq \sum_{i=1}^{m} V_{i}
$$

where $\sum$ stands for the boolean sum. This implies $\sum_{i=1}^{m} V_{i}+V=\sum_{i=1}^{m} V_{i}$, which contradicts the fact that $K$ is an $\ell$-superimposed code. 
With the use of projective planes, we can prove that, in the case where $\ell$ is a prime power, there exist connected graphs admitting $(1, \leq \ell)$-identifying codes of cardinality $\Theta\left(\ell^{2}\right)$. We recall that a projective plane of order $n$ is an hypergraph on $n^{2}+n+1$ vertices such that :

- Any pair of vertices lie in a unique hyperedge,

- Any two hyperedges have a unique common vertex,

- Every vertex is contained in $n+1$ hyperedges, and

- Every hyperedge contains $n+1$ vertices.

Note that some of these properties are redundant. We denote $\mathbb{P}_{n}$ the projective plane of order $n$. It is known that $\mathbb{P}_{n}$ exists if $n$ is the power of a prime number. Projective planes of order $n$ are also known as $2-\left(n^{2}+n+1, n+1,1\right)$ designs, or $S\left(2, n+1, n^{2}+n+1\right)$ Steiner systems.

Lemma 4 If $q$ is a prime power, then there exists a connected graph $G_{q}$ on $2\left(q^{2}+q+1\right)$ vertices admitting a $(1, \leq q)$-identifying code. Moreover, $G_{q}$ is $(q+1)$-regular.

Proof : Assume that $q$ is a prime power, and consider a finite projective plane $\mathbb{P}_{q}$ of order $q$. In other words, we have a $\left(q^{2}+q+1\right)$-element set $S$ and $\mathbb{P}_{q}$ consists of $q^{2}+q+1$ hyperedges, each hyperedge being a $(q+1)$-element subset of $S . \mathbb{P}_{q}$ has the property that every pair of elements of $S$ is contained in a unique hyperedge. The number of hyperedges is $q^{2}+q+1$; each element of $S$ is contained in exactly $q+1$ hyperedges; and, finally, every two hyperedges have exactly one element in common.

Denote by $A$ the adjacency matrix of $\mathbb{P}_{q}$, where the rows are labelled by the elements of $S$ and the columns by the hyperedges, and the entry $A_{i j}$ is 1 if the $i$-th element is in the $j$-th hyperedge, and 0, otherwise. (By labelling the elements and hyperedges suitably, we could make $A$ symmetric, but we do not need it here.) Now, every row (resp. column) of $A$ has exactly $q+1$ ones; and every two rows (resp. every two columns) of $A$ have exactly one 1 in common.

We now use $A$ to construct a graph $G_{q}$ as follows. Let

$$
B=\left(\begin{array}{ll}
0 & A \\
A^{T} & 0
\end{array}\right),
$$

and let $G_{q}$ be the simple, non-oriented graph whose adjacency matrix is $B$, i.e. vertices $i$ and $j$ are adjacent in $G_{q}$ if and only if $B_{i j}=1$. The graph $G_{q}$ is well-defined since $B$ is a symmetric matrix having only 0's on its diagonal.

Obviously, the graph $G_{q}$ has $2\left(q^{2}+q+1\right)$ vertices and is $(q+1)$-regular. Moreover, $G_{q}$ is bipartite, as all the edges go between the first $q^{2}+q+1$ and the last $q^{2}+q+1$ vertices. Clearly, $G_{q}$ is connected: Given any two of the first $q^{2}+q+1$ vertices, there is a unique vertex among the last $q^{2}+q+1$ vertices which is connected to both of them, and the connectivity easily follows. 
Moreover, we can prove that the whole vertex set is a $(1, \leq q)$-identifying code of $G_{q}$. Assume that $X$ is a subset of the vertex set having at most $q$ elements. Assume further that we do not know $X$, but that we know $I(X)$. Let $v$ be an arbitrary vertex. Clearly $|I(v)|=q+2$, and

For every vertex $u \neq v$, the set $I(u)$ contains at most one element of $I(v) \backslash\{v\}$.

(Remark that we can obtain the identifying sets of individual vertices by changing all the diagonal elements of $B$ into 1's: We get a matrix $B^{\prime}$ where the $i$-th row gives the identifying set of the $i$-th vertex.) For the vertices $u$ in the same part of the bipartition as $v,(2)$ follows from the properties of projective planes; and for the other vertices (2) is trivial by construction. Consequently, if $v \in X$, then all the $q+2$ elements of $I(v)$ are in $I(X)$; but if $v \notin X$, then at most $q+1$ elements of $I(v)$ are in $I(X)$. So, we can immediately tell by looking at $I(X)$, whether $v$ is in $X$ or not; and this is true for all $v \in X$, completing the proof.

Finally, we need the following :

Lemma 5 Let $C$ be $a(1, \leq \ell)$-identifying code of a graph $G$, and let $X$ and $Y$ be distinct subsets of at most $\ell$ vertices of $G$. Then we have either

$$
|X|+|I(X) \Delta I(Y)|>\ell \quad \text { or } \quad|Y|+|I(X) \Delta I(Y)|>\ell .
$$

Proof : Let $X^{\prime}:=X \cup I(X) \Delta I(Y)$ and $Y^{\prime}:=Y \cup I(X) \Delta I(Y)$. It is easy to see that $I\left(X^{\prime}\right) \Delta I\left(Y^{\prime}\right)=\emptyset$. Since $C$ is a $(1, \leq \ell)$-identifying code, this implies $\left|X^{\prime}\right|>\ell$ or $\left|Y^{\prime}\right|>\ell$.

Now we are ready to prove the validity of the construction described in the previous section.

Proof of Theorem 2 : The case $\ell=1$ is already known [9], and derive from the case $\ell=2$. Now let $\ell \geq 2$. Let $N=\left\lceil\ell^{2} \log n\right\rceil$ and let $K$ be a maximal $\ell$-superimposed code of $\{0,1\}^{N}$. By Theorem 1 we know that there exists such a $K$ satisfying $|K| \geq \Omega(n)$. Let $M$ be the matrix whose columns are the vectors of $K$. In Step 2 of the construction we need to find an $N \times N$ submatrix $M^{\prime}$ of $M$ having a 1 on each one of its rows : since $K$ is maximal, then by Lemma 2 such a submatrix exists. In Step 3 of the construction we need a graph $H$ having a $(1, \leq \ell)$-identifying code. If $\ell$ is a prime power then we take $H=G_{\ell}$ as constructed in Lemma 4. If $\ell$ is not a prime power, then by Bertrand's Conjecture proved in 1850 by Chebyshev and later by Erdös in his first paper [5] - we know that there exists a prime number $p$ in the interval $[\ell, 2 \ell]$, and we take $H=G_{p}$ as constructed in Lemma 4. Since $p \geq \ell$, then $G_{p}$ admits a $(1, \leq p)$-identifying code implies that $G_{p}$ admits a $(1, \leq \ell)$-identifying code. Both $H=G_{\ell}$ and $H=G_{p}$ have $\Theta\left(\ell^{2}\right)$ vertices.

Now let $\mathcal{G}$ and $C$ be as constructed in Step 3 of the construction. We prove that $C$ is a $(1, \leq \ell)$-identifying code of $\mathcal{G}$. Let $X$ and $Y$ be two subsets of vertices of $\mathcal{G}$ of cardinality less or equal to $\ell$. We show that $I(X)=I(Y)$ if and only if $X=Y$. We proceed in 
two steps: first we prove that $I(X)=I(Y) \Rightarrow X \cap C=Y \cap C$, and then we prove that $I(X)=I(Y) \Rightarrow X \backslash C=Y \backslash C$. In the rest of the proof, we assume that $I(X)=I(Y)$.

(a) By way of contradiction, let us assume that $X \cap C \neq Y \cap C$, and let $H_{i}$ be a connected component of $\mathcal{G}[C]$ on which $X$ and $Y$ differ. Denoting $X_{i}=X \cap H_{i}$ and $Y_{i}=Y \cap H_{i}$, we have $X_{i} \neq Y_{i}$. Since $H_{i} \subset C$ and $V\left(H_{i}\right)$ is a $(1, \leq \ell)$-identifying code of $H_{i}$, then we have $I\left(X_{i}\right) \neq I\left(Y_{i}\right)$. If there is an $h \in H_{i}, h \neq h_{i}$, such that $h \in I\left(X_{i}\right) \Delta I\left(Y_{i}\right)$, then we obtain a contradiction since $h \notin N\left(X \backslash X_{i}\right) \cup N\left(Y \backslash Y_{i}\right)$ : the neighborhood of $h \neq h_{i}$ is contained in $H_{i}$, and consequently $h \in I\left(X_{i}\right) \Delta I\left(Y_{i}\right) \Rightarrow h \in I(X) \Delta I(Y)$. Hence $I\left(X_{i}\right) \Delta I\left(Y_{i}\right)=\left\{h_{i}\right\}$. By Lemma 5 we may assume that $\left|X_{i}\right|=\ell$, that is to say $X=X_{i} \subseteq H_{i}$ and $h_{i} \in I(X) \backslash I\left(Y_{i}\right)$. Since our assumption is that $I(X)=I(Y)$, it means that there exists a neighbor $y$ of $h_{i}$ belonging to $Y \backslash C$. By Lemma 3, $y$ is neighbor of at least $\ell$ vertices of $C$ (remember that to each column vector $W$ of $M-M^{\prime}$ we associated a vertex $\phi(W)$ which is neighbor to $h_{i}$ for all $i$ such that the $i$-th coordinate of $W$ is 1). Since $\ell \geq 2$, then there exists $h_{j} \in C, h_{j} \neq h_{i}$, such that $h_{j} \in I(Y) \backslash I(X)$ : this contradicts $I(X)=I(Y)$.

(b) Set $X^{\prime}=X \backslash C$ and $Y^{\prime}=Y \backslash C$. Assume that $X^{\prime} \neq Y^{\prime}$. Now, to each $h_{i} \in$ $I\left(X^{\prime}\right) \Delta I\left(Y^{\prime}\right)$, we can associate a unique $h_{i}^{\prime} \in X \cap C=Y \cap C$. Indeed, since $I(X)=I(Y)$, then for each $h_{i}$ in, say, $I\left(X^{\prime}\right) \backslash I\left(Y^{\prime}\right)$, there exists an $h_{i}^{\prime} \in Y \cap H_{i}=X \cap H_{i}$ such that $h_{i} \in N\left(h_{i}^{\prime}\right)$. Hence there exists an injection $I\left(X^{\prime}\right) \Delta I\left(Y^{\prime}\right) \hookrightarrow X \cap C=Y \cap C$. This shows that :

$$
|X| \geq\left|X^{\prime}\right|+\left|I\left(X^{\prime}\right) \Delta I\left(Y^{\prime}\right)\right| \quad \text { and } \quad|Y| \geq\left|Y^{\prime}\right|+\left|I\left(X^{\prime}\right) \Delta I\left(Y^{\prime}\right)\right|
$$

Now, remind that $X^{\prime}=\left\{v_{p}\right\}_{p \in P}$ and $Y^{\prime}=\left\{v_{q}\right\}_{q \in P}$ correspond to two different sets $\phi^{-1}(X)=\left\{V_{p}\right\}_{p \in P}$ and $\phi^{-1}(Y)=\left\{V_{q}\right\}_{q \in Q}$ of column vectors of the matrix $M \backslash M^{\prime}$. Note that $\left|I\left(X^{\prime}\right) \Delta I\left(Y^{\prime}\right)\right|$ is the number of coordinates on which $\sum_{p \in P} V_{p}$ and $\sum_{q \in Q} V_{q}$ differ, where $\sum$ stands for the boolean sum. Let $\mathcal{I}$ denote the set of coordinates on which $\sum_{p \in P} V_{p}$ and $\sum_{q \in Q} V_{q}$ differ: $|\mathcal{I}|=\left|I\left(X^{\prime}\right) \Delta I\left(Y^{\prime}\right)\right|$. Now, for each coordinate $i \in \mathcal{I}$, let $W_{\tau(i)}$ be a column vector of $M^{\prime}$ having its $i$-th coordinate equal to 1 . By definition of the $W_{\tau(i)}$ 's, we have :

$$
\sum_{p \in P} V_{p}+\sum_{i \in \mathcal{I}} W_{\tau(i)}=\sum_{q \in Q} V_{q}+\sum_{i \in \mathcal{I}} W_{\tau(i)} .
$$

Since $M$ is the matrix of an $\ell$-superimposed code, this implies that:

$$
|P|+|\mathcal{I}|>\ell \quad \text { or } \quad|Q|+|\mathcal{I}|>\ell .
$$

Recalling (3), since $|P|=\left|X^{\prime}\right|,|Q|=\left|Y^{\prime}\right|$, and $|\mathcal{I}|=\left|I\left(X^{\prime}\right) \Delta I\left(Y^{\prime}\right)\right|$, we obtain:

$$
|X|>\ell \quad \text { or } \quad|Y|>\ell
$$

which is a contradiction.

Hence $C$ is a $(1, \leq \ell)$-identifying code of $\mathcal{G}$. $C$ has cardinality $N \times|H|$, and $\mathcal{G}$ has $N \times|H|+(|K|-N)$ vertices. Since $N=\left\lceil\ell^{2} \log n\right\rceil,|K| \geq \Omega(n)$ and $|H|=\Theta\left(\ell^{2}\right)$, then we have

$$
|C|=\Theta\left(\ell^{2}\right)\left\lceil\ell^{2} \log n\right\rceil \quad \text { and } \quad|\mathcal{G}|=\Omega(n)
$$

hence

$$
|C|=O\left(\ell^{4} \log |\mathcal{G}|\right)
$$




\section{Conclusion}

In this paper we showed a correspondence between $(1, \leq \ell)$-identifying codes and $\ell$ superimposed codes, which enabled us to construct a $(1, \leq \ell)$-identifying code of cardinality $O\left(\ell^{4} \log n\right)$ in a graph on $n$ vertices from a maximal $\ell$-superimposed code of length $\left\lceil\ell^{2} \log n\right\rceil$. This answers a question of $[6]$.

Our method can be used to answer another interesting question. In [12] it is shown that a graph admitting a $(1, \leq \ell)$-identifying code has its minimum degree greater or equal to $\ell$. We wondered if there existed graphs admitting a $(1, \leq \ell)$-identifying code with minimum degree equal to $\ell$. The idea of the construction of Section 2 can be used to answer this question : take $\ell$ copies $H_{1}, \ldots, H_{\ell}$ of a connected graph $H$ admitting a $(1, \leq \ell$ )-identifying code (from Lemma 4 we know that such an $H$ exists), specify $\ell$ vertices $h_{i} \in H_{i}$ for $i=1, \ldots, \ell$ and then construct a graph $\mathcal{G}^{\prime}$ by joining the $H_{i}$ 's with a new vertex $u$ such that $u h_{i}$ is an edge of $\mathcal{G}^{\prime}$ for all $i=1, \ldots, \ell$. It is easy to see that $\mathcal{G}^{\prime}$ is a graph admitting a $(1, \leq \ell)$-identifying code. Indeed, let $X$ and $Y$ be two distinct subsets of at most $\ell$ vertices of $\mathcal{G}^{\prime}$. If $u \notin X \cup Y$, then clearly $N[X] \neq N[Y]$ since $H$ admits a $(1, \leq \ell)$-identifying code. If $u \in X \cap Y$, then let $i$ be such that $X \cap H_{i}=: X_{i} \neq Y_{i}:=Y \cap H_{i}$. As $|X i| \leq \ell-1$ and $|Y i| \leq \ell-1$, then by Lemma 5 we know that $|N[X i] \Delta N[Y i]| \geq 2$. Since $u$ has only one neighbor $h_{i}$ in $H_{i}$, then $N[X] \neq N[Y]$. Finally, if, say, $u \in X \backslash Y$ , then $Y$ has to have a nontrivial intersection with each copy $H_{1}, \ldots, H_{\ell}$. Hence $|Y|=\ell$ and for all $i=1, \ldots, \ell$ we have $\left|Y \cap H_{i}\right|=1$. Since $H$ admits a $(1, \leq \ell)$-identifying code then $\delta(H) \geq \ell \geq 1$ and then $\left|N[Y] \cap H_{i}\right| \geq 2$ for all $i=1, \ldots, \ell$. This implies that for all $i=1, \ldots, \ell$ there exists an $x_{i} \in X \cap H_{i}$. Since $X$ contains also $u$, this contradicts $|X| \leq \ell$.

Thus, we proved the following :

Proposition 1 For all $\ell \geq 1$ there exists a graph $G_{\ell}$ admitting a $(1, \leq \ell)$ - identifying code with minimum degree equal to $\ell$.

We wonder if there exists $\ell$-regular graphs admitting $(1, \leq \ell)$-identifying codes. Remind that Lemma 4 says that, if $\ell$ is a prime power, then there exists $(\ell+1)$-regular graphs admitting a $(1, \leq \ell)$-identifying code.

We recall from $[6]$ that a $(1, \leq \ell)$-identifying code of a graph on $n$ vertices has a

cardinality greater or equal to $\Omega\left(\frac{\ell^{2}}{\log \ell} \log n\right)$. This is a direct consequence of Theorem 1 . Here we showed how to construct graphs having a $(1, \leq \ell)$-identifying code of cardinality $O\left(\ell^{4} \log n\right)$. Our construction is based on the existence of connected graphs on $\Theta\left(\ell^{2}\right)$ vertices admitting a $(1, \leq \ell)$ - identifying code (Lemma 4$)$. If we could improve Lemma 4 by constructing graphs on less than $\Theta\left(\ell^{2}\right)$ vertices admitting a $(1, \leq \ell)$-identifying code, then this would directly result in an improvement of Theorem 2.

Hence the minimum number of vertices of a connected graph admitting a $(1, \leq \ell)$ identifying code is an interesting question, that we pose here as an open problem. 


\section{Acknowledgment}

The authors would like to thank the anonymous referee, who made very helpful comments and suggested the use of projective planes to construct a graph on $\Theta\left(\ell^{2}\right)$ vertices admitting a $(1, \leq \ell)$-identifying code (Lemma 4$)$. This resulted in a significant improvement of our main result (Theorem 2 ).

\section{References}

[1] U. Blass, I. Honkala, S. Litsyn, On Binary Codes for Identification, Journal of Combinatorial Designs 8 (2000), 151-156

[2] U. Blass, I. Honkala, S. Litsyn, Bounds on Identifying Codes, Discrete Mathematics 241 (2001), 119-128.

[3] G. Cohen, I. Honkala, S. Litsyn, A. Lobstein, Covering Codes, Elsevier, NorthHolland Mathematical Library (1997).

[4] A. G. D'yachkov, V. V. Rykov, Bounds on the length of disjunctive codes, Problems of Information Transmission 18 (1983), 166-171.

[5] P. Erdös, Beweis eines Satzes von Tschebyschef, Acta Litterarum ac Scientiarum, Szeged 5 (1932), 194-198.

[6] A. Frieze, R. Martin, J. Moncel, M. Ruszinkó, C. Smyth, Codes Identifying Sets of Vertices in Random Networks, submitted.

[7] I. Honkala, T. Laihonen, S. Ranto, On Codes Identifying Sets of Vertices in Hamming Spaces, Designs, Codes and Cryptography 24(2) (2001), 193-204.

[8] F. K. Hwang, V. Sós, Non-adaptive hypergeometric group testing, Studia Scientiarum Mathematicarum Hungaricae 22(1-4) (1987), 257-263.

[9] M. G. Karpovsky, K. Chakrabarty, L. B. Levitin, On a New Class of Codes for Identifying Vertices in Graphs, IEEE Transactions on Information Theory 44(2) (1998), 599-611.

[10] M. G. Karpovsky, K. Chakrabarty, L. B. Levitin, D. R. Avreky, On the Covering of Vertices for Fault Diagnosis in Hypercubes, Information Processing Letters, 69 (1999), 99-103.

[11] W. H. Kautz, R. R. Singleton, Nonrandom binary superimposed codes, IEEE Transformations on Information Theory 10(4) (1964), 363-377.

[12] T. Laihonen, S. Ranto, Codes Identifying Sets of Vertices, Lecture Notes in Computer Science 2227 (2001), 82-91.

[13] M. Ruszinkó, On the upper bound of the size of the r-cover-free families, Journal of Combinatorial Theory Series A 66(2) (1994), 302-310.

[14] http://www.infres.enst.fr/ lobstein/debutBIBidetlocdom.ps 